\title{
LVIII. Inverse interpolation by means of a reversed series
}

\section{C.E. Van Orstrand}

To cite this article: C.E. Van Orstrand (1908) LVIII. Inverse interpolation by means of a reversed series, Philosophical Magazine Series 6, 15:89, 628-638, DOI: 10.1080/14786440809463804

To link to this article: http://dx.doi.org/10.1080/14786440809463804

Published online: 16 Apr 2009.

Submit your article to this journal \lceil

Џll Article views: 2

Q View related articles $\square$ 
LVIII. Inverse Interpolation by Means of a Reversed Series. By C. E. Van Orstrand *.

THE formulas developed by Newton, Bessel, and Stirling for the direct interpolation of a value of a function from values tabulated at equal intervals of the argument, are considered to be among the most important of their contributions to the science of applied mathematics. The converse problem, that of finding the argument when a value of the function is given, although of about equal importance, seems not to have received the thorough treatment which mathematicians have given to the subject of direct interpolation. Apart from methods applicable only to special cases, there are two general methods $\dagger$ now in use which are to a certain extent satisfactory. Each of the methods is in reality based upon the same principle-that of diminishing the interval of interpolation. In the one case this is accomplished by computing values of the function at equal intervals of the argument for values preceding and following the required value. If the interval of the new series of tabular values is sufficiently small, the correct argument can be found by taking second differences into account. In the second case, the function and its first and second derivatives are computed for a value of the argument $(n)$ true to the nearest tenth of a unit. It is then generally sufficient to write the interpolation formula as a Taylor's series involving only the first and second derivatives. The correction to the approximate value of the argument is then found by reverting this series of two terms. Each of the methods has the serious disadvantage of being a tentative process, and neither of them provides a satisfactory check on the computation, without the aid of additional quantities.

As a means of avoiding the difficulties noted above, it is desirable to call attention to the use which may be made of a reverted series as a formula for inverse interpolation. To derive this formula, let the tabular values of the function $\left(\mathrm{F}_{-3}, \mathrm{~F}_{-2}, \ldots\right)$ and its successive differences (the $a^{2} \mathrm{~s}, b$ 's ...) be represented by the following schedule :-

* Communicated by the Author.

† Rice, 'Theory and Practice of Interpolation,' pp. 192-5. 
Inverse Interpolation by Means of a Reversed Series. 629

\begin{tabular}{|c|c|c|c|c|c|c|}
\hline $\mathbf{T}$ & $\mathbf{F}\left(\mathrm{I}^{\prime}\right)$ & $\Delta^{\prime}$ & $\Delta^{\prime \prime}$ & $\Delta^{\prime \prime \prime}$ & $\Delta^{\mathrm{IV}}$ & $\Delta^{\mathrm{v}}$ \\
\hline$t-3 \omega$ & $\mathrm{F}_{-3}$ & $a_{-3}$ & $b_{-2}$ & $c_{-2}$ & $d_{-1}$ & $e_{-1}$ \\
$t-2 \omega$ & $\mathrm{F}_{-2}$ & $a_{-2}$ & $b_{-1}$ & $c_{-1}$ & $d_{0}$ & $e_{1}$ \\
$t-\omega$ & $\mathbf{F}_{-1}$ & $a_{-1}$ & $b_{0}$ & $c_{1}$ & $d_{1}$ & \\
$t$ & $\mathrm{~F}_{0}$ & $a_{1}$ & $b_{1}$ & $c_{2}$ & & \\
$t+\omega$ & $\mathbf{F}_{1}$ & $a_{2}$ & $b_{2}$ & & & \\
$t+2 \omega$ & $\mathrm{F}_{2}$ & $a_{3}$ & & & & \\
\hline
\end{tabular}

Put also

$$
\begin{array}{rlrl}
a & =\frac{1}{2}\left(a_{1}+a_{-1}\right) & a^{\prime} & =a-\frac{c}{6}+\frac{e}{30} \\
c & =\frac{1}{2}\left(c_{1}+c_{-1}\right) & b^{\prime} & =\frac{b_{0}}{2}-\frac{d_{0}}{24} \\
e & =\frac{1}{2}\left(e_{1}+e_{-1}\right) & c^{\prime} & =\frac{c}{6}-\frac{e}{24} \\
\Delta \mathrm{F} & =\mathrm{F}_{n}-\mathrm{F}_{0} & d^{\prime} & =\frac{d_{0}}{24} \\
e^{\prime} & =\frac{e}{120 .}
\end{array}
$$

Then, using Stirling's expression for the successive derivatives, because of their simplicity and rapid convergence, and writing the interpolation formula as a Taylor's series,

$$
\Delta \mathrm{F}=a^{\prime} n+b^{\prime} n^{2}+c^{\prime} n^{3}+d^{\prime} n^{4}+e^{\prime} n^{5}+\ldots . . .
$$

The solution of the problem consists in finding the value of $n$ when $\Delta \mathrm{F}, a^{\prime}, b^{\prime}, c^{\prime}, \ldots$ are given. To accomplish this, we revert equation (1) by means of the usual method of equating coefficients, or by means of the expression obtained by Professor McMahon for the general term of a reverted series *, and obtain

$$
\begin{aligned}
n & =\frac{\Delta \mathrm{F}}{a^{\prime}}-\frac{b^{\prime}}{a^{\prime}}\left(\frac{\Delta \mathrm{F}}{a^{\prime}}\right)^{2}+\left[2\left(\frac{b^{\prime}}{a^{\prime}}\right)^{2}-\frac{c^{\prime}}{a^{\prime}}\right]\left(\frac{\Delta \mathrm{F}}{a^{\prime}}\right)^{3} \\
& +\left[5 \frac{b}{a^{\prime}} \frac{c}{}^{\prime}-\frac{d^{\prime}}{a^{\prime}}-5\left(\frac{b^{\prime}}{a^{\prime}}\right)^{3}\right]\left(\frac{\Delta \mathrm{F}}{a^{\prime}}\right)^{4} \\
& +\left[3\left(\frac{c^{\prime}}{a^{\prime}}\right)^{2}+6 \frac{b^{\prime}}{a^{\prime}} \frac{d^{\prime}}{a^{\prime}}-\frac{e^{\prime}}{a^{\prime}}-21\left(\frac{b^{\prime}}{a^{\prime}}\right)^{2} \frac{c^{\prime}}{a^{\prime}}+14\left(\frac{b^{\prime}}{a^{\prime}}\right)^{4}\right]\left(\frac{\Delta \mathrm{F}}{a^{\prime}}\right)^{5}+\ldots
\end{aligned}
$$

* Bull. Am. Math. Soc. iii. p. 170 (1893-1894).

Phil. Mag. S. 6. Vol. 15. No. 89. May 1908. 
Equation (2) may be put into a more convenient form for computation by rearranging the terms and putting $n_{1}=\Delta \mathrm{F} \div a^{\prime}$ and $r=n_{1} \div a^{\prime}$. Then

$$
\begin{aligned}
n=n_{1} & +\left[-r b^{\prime}+2\left(r b^{\prime}\right)^{2}-5\left(r b^{\prime}\right)^{3}+14\left(r b^{\prime}\right)^{4}\right] n_{1} \\
& +\left[r c^{\prime}\left(-1+5 r b^{\prime}-21\left(r b^{\prime}\right)^{2}\right] n_{1}{ }^{2}\right. \\
& +\left\lfloor r d^{\prime}\left(-1+6 r b^{\prime}\right)+3\left(r c^{\prime}\right)^{2}\right] n_{1}^{3} \\
& +\left[-r e^{\prime}\right] n_{1}{ }^{4}+\ldots .
\end{aligned}
$$

Now substitute

$$
\begin{aligned}
& f_{1}=-r b^{\prime}+2\left(r b^{\prime}\right)^{2}-5\left(r b^{\prime}\right)^{3}+14\left(r b^{\prime}\right)^{4} \\
& f_{2}=r c^{\prime}\left[-1+5 r b^{\prime}-21\left(r b^{\prime}\right)^{2}\right] \\
& f_{3}=r d^{\prime}\left\lceil-1+6 r b^{\prime}\right]+3\left(r c^{\prime}\right)^{2} \\
& f_{4}=-r e^{\prime}
\end{aligned}
$$

in (3), and the equation for inverse interpolation, inclusive of fifth differences, is

$$
n=n_{1}+f_{1} n_{1}+f_{2} n_{1}^{2}+f_{3} n_{1}^{3}+f_{4} n_{1}^{4} .
$$

For backward interpolation $n_{1}$ is negative. A slightly different form of (4) convenient for logarithmic computation is

$$
n=n_{1}+f_{1} n_{1}+r c^{\prime} f_{2}^{\prime} n_{1}^{2}+r d^{\prime} f_{3}^{\prime} n_{1}{ }^{2}+3\left(r c^{\prime}\right)^{2} n_{1}{ }^{3}+f_{4} n_{1}{ }^{4} \ldots
$$
in which

$$
\begin{aligned}
& f_{2}^{\prime}=-1+5 r b^{\prime}-21\left(r b^{\prime}\right)^{2} \\
& f_{3}^{\prime}=-1+6 r b^{\prime} .
\end{aligned}
$$

The expressions for the $f^{\prime}$ 's, as obtained by developing the reverted series to the same order of powers as the given series, are represented by the following groups, according as each series terminates with the second, third, or fourth power :

$$
\begin{array}{ll}
f_{1} \equiv-r b^{\prime} \equiv-r b^{\prime}+2\left(r b^{\prime}\right)^{2} & \equiv-r b^{\prime}+2\left(r b^{\prime}\right)^{2}-5\left(r b^{\prime}\right)^{3} \\
f_{2} \equiv \equiv-r c^{\prime} & \equiv r c^{\prime}\left(-1+5 r b^{\prime}\right) \\
f_{3} \equiv & \equiv-r d^{\prime} .
\end{array}
$$

Analytically, there are an infinite number of the $f$ 's and each contains an infinite number of terms. The number of terms required in any particular reversion is easily ascertained from the data. As a complete formula for inverse interpolation, applicable in all cases, the reversed series is unfortunately not always sufficiently convergent when a limited number of terms is used. However, the most important applications of reversion obtain when the numerical magnitudes of the successive orders of differences gradually diminish. It will be found for such functions that $r b^{\prime}<0 \cdot 1$, and since $n<\frac{1}{2}$, the 
Interpolation by Means of a Reversed Series.

doubly infinite series will always converge ${ }^{*}$. The cases in which the series fails to converge with sufficient rapidity are therefore exceptional and are easily discovered. In fact it is only necessary to see if the terms at the end of each horizontal series, such as $14\left(r b^{\prime}\right)^{4} n_{1}, 21 r c^{\prime}\left(r b^{\prime}\right)^{2} n^{2}, \ldots$, are small in comparison with $n_{1}$.

The quantities $\left(a^{\prime} b^{\prime} c^{\prime} d^{\prime} e^{\prime}\right)$ are easily computed when the first derivatives are tabulated. Representing the functional values, the first derivatives, and the successive differences of the first derivatives by the scheme:

\begin{tabular}{|c|c|c|c|c|c|c|}
\hline $\mathrm{T}$ & $\mathrm{F}(\mathrm{T})$ & $\omega \mathrm{F}^{\prime \prime}$ & $\Delta^{\prime}$ & $\Delta^{\prime \prime}$ & $\Delta^{\prime \prime}$ & $\Delta^{\mathrm{IV}}$ \\
\hline$t-3 \omega$ & $\mathrm{F}_{-3}$ & $a_{-3}$ & $\hat{\beta}_{-3}$ & & & \\
$t-2 \omega$ & $\mathrm{F}_{-2}$ & $a_{-2}$ & $\beta_{-2}$ & $\gamma_{-2}$ & & \\
$t-\omega$ & $\mathrm{F}_{-1}$ & $a_{-1}$ & $\beta_{-1}$ & $\gamma^{-1}$ & $\delta_{-2}$ & $\epsilon_{-1}$ \\
$t$ & $\mathrm{~F}_{0}$ & $a_{0}$ & $\beta_{1}$ & $\gamma_{0}$ & $\delta_{-1}$ & $\epsilon_{0}$ \\
$t+\omega$ & $\mathrm{F}_{1}$ & $a_{1}$ & $\beta_{2}$ & $\gamma_{1}$ & $\delta_{1}$ & $\epsilon_{1}$ \\
$t+2 \omega$ & $\mathrm{F}_{2}$ & $a_{2}$ & $\beta_{3}$ & $\gamma_{2}$ & $\delta_{2}$ & \\
$t+3 \omega$ & $\mathrm{F}_{3}$ & $a$ & & & & \\
\hline
\end{tabular}

these auxiliary quantities are represented by the simple forms :-

$$
\begin{array}{ll}
a^{\prime}=\alpha_{0} & \\
b^{\prime}=\frac{\beta}{2}-\frac{\delta}{12} & \\
c^{\prime}=\frac{\gamma_{0}}{6}-\frac{\epsilon_{0}}{72} & \beta=\frac{1}{2}\left(\beta_{1}+\beta_{-1}\right)=\beta_{1}-\frac{\gamma_{0}}{2} \\
d^{\prime}=\frac{\delta}{24} & \delta=\frac{1}{2}\left(\delta_{1}+\delta_{-1}\right)=\delta_{1}-\frac{\delta_{0}}{2}
\end{array}
$$

when Stirling's formulas for the successive derivatives are used. Evidently,

$$
b^{\prime}=\frac{\omega^{2} \mathrm{~F}_{0}^{\prime \prime}}{2} ; c^{\prime}=\frac{\omega^{3} \mathrm{~F}_{0}{ }^{\prime \prime \prime}}{6} ; d^{\prime}=\frac{\omega^{4} \mathrm{~F}_{0}^{\mathrm{iv}}}{24} ; e^{\prime}=\frac{\omega^{5} \mathrm{~F}_{0}{ }^{\mathrm{v}}}{120} .
$$

The computation of $a^{\prime}, b^{\prime}, c^{\prime}, d^{\prime}, e^{\prime} \ldots$ can be checked in various ways. In general it is quite sufficient to duplicate the work. An independent check is of course obtained by computing

* Harkness \& Morley, 'Treatise on Theory of Functions,'p. 116. 
632

Mr. C. E. Van Orstrand : Inverse

the derivatives from the formulas of either Newton or Bessel, preferably the latter for arguments other than at the beginning or end of a series of tabular values.

As an application of formula (4) let it be required to find the interpolation interval $(n)$ from the following tabular values of the function and its differences, when the logarithm of Mercury's distance from the Earth equals $\mathrm{F}_{n}=9 \cdot 7968280$.

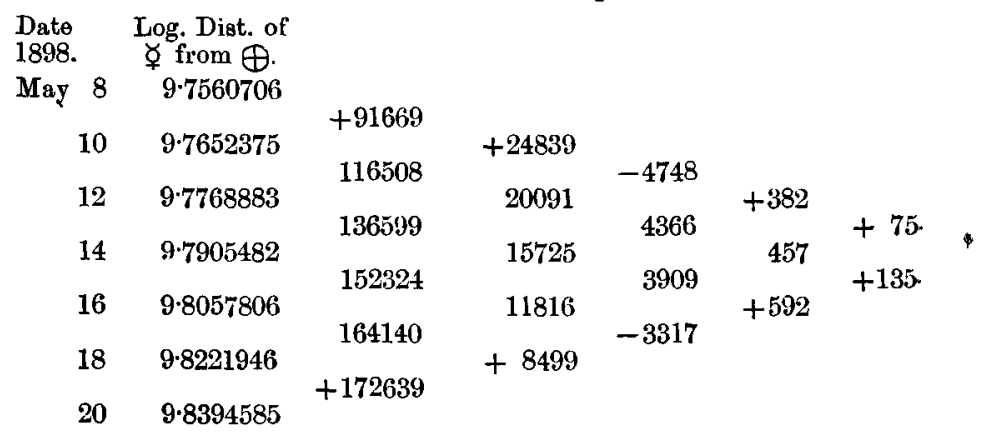

We take from the table,

$$
\begin{aligned}
& \mathrm{F}_{0}=9 \cdot 7905482, \\
& a=\frac{1}{2}\left(a_{1}+a_{-1}\right)=+144461 \cdot 5 \\
& b_{0}=+15725 \\
& c^{\circ}=\frac{1}{2}\left(c_{1}+c_{-1}\right)=-4137 \cdot 5 \\
& d_{0}=+\quad 457 \\
& e^{=}=\frac{1}{2}\left(e_{1}+e_{-1}\right)=+\quad 105 \cdot 0
\end{aligned}
$$

and then compute,

$$
\begin{aligned}
& b^{\prime}=\frac{b_{0}}{2}-\frac{d_{0}}{24} \quad=+7843.5 \quad 3.894510 \quad r b^{\prime}=+0.233773 \\
& c^{\prime}=\frac{c}{6}-\frac{e}{24} \quad=-\quad 693.9 \quad 2 \cdot 84130 n \quad r c^{\prime}=-0.0020681 \\
& d^{\prime}=\frac{d_{0}}{24} \quad=+\quad 19 \cdot 0 \quad 1.2788 \quad r d^{\prime}=+0.0000566 \\
& e^{\prime}=\frac{e}{120} \quad=+\quad 0.9 \quad 9.954_{-10} \quad r e^{\prime}=+0.0000027 \\
& \Delta \mathrm{F}=\mathrm{F}_{n}-\mathrm{F}_{0} \quad=+62798 \cdot 0 \quad 4 \cdot 7979458 \quad f_{1}=-0.0223441 \\
& a^{\prime}=a-\frac{e}{6}+\frac{e}{30}=+145154 \cdot 6 \quad 5 \cdot 1618308 \quad f_{2}=+0 \cdot 0018500 \\
& n_{1}=\Delta \mathrm{F} \div a^{\prime}=\quad 9 \cdot 6361150_{-10} \quad f_{3}=+0 \cdot 0000359 \\
& r=n_{1} \div a^{\prime} \quad=\quad 4 \cdot 4742842_{-10} \quad f_{4}=-0.0000027 \text {. }
\end{aligned}
$$


Substituting in equation (4) we compute $n$; then substituting $n$ in equation (1) we compute $\Delta \mathrm{F}$ as a check. Thus,

$$
\begin{array}{rlr}
n_{1} & =+0 \cdot 4326284 & a^{\prime} n=+61444 \cdot 7 \\
f_{1} n_{1}=-0 \cdot 0096667 & b^{\prime} n^{2}=+1405 \cdot 4 \\
f_{2} n_{1}^{2}=+0 \cdot 0003463 & e^{\prime} n^{3}=- & 52 \cdot 6 \\
f_{3} n_{1}^{3}=-0 \cdot 0000029 & d^{\prime} n^{4}=+ & 0 \cdot 6 \\
f_{4} n_{1}^{4}=-0 \cdot 0000001 & e^{\prime} n^{5}= & 0 \cdot 0 \\
n=\Sigma & =+0 \cdot 423305 & \Delta \mathrm{F}=\Sigma=+62798 \cdot 1 .
\end{array}
$$

By means of the second method of inverse interpolation previously explained, Rice* finds $n=0 \cdot 423303$. This discrepancy is due to neglecting units in the seventh decimal place. The value of $\Delta \mathrm{F}$ computed from this value of $n$ and Stirling's derivatives is $\Delta \mathrm{F}=62797 \cdot 7$.

There are a great number of tabulated functions in which second differences are approximately constant and in which the term $2 n_{1}\left(r b^{\prime}\right)^{2}$ is negligible. In such cases the following rule applies: Divide the increment of the function $(\Delta \mathrm{F})$ by the mean of the first differences $\left(a_{1}+\frac{1}{2} b_{0}\right)$, and likewise divide this quotient $\left(n_{1}\right)$ by the same quantity; then the product of these two quotients $\left(n_{1} r\right)$ and one-half the second difference $\left(\frac{1}{2} b_{0}\right)$ is the correction to be applied algebraically to the first quotient $\left(n_{1}\right)$ in order to obtain the required interpolation interval $(n)$. The algebraic signs of the quantities need not be taken into account; for it is plain that if the numerical values of the first differences are increasing, the quotient $\left(n_{1}\right)$ is too large and the correction is therefore negative, while if the numerical values of the first differences are decreasing, the value of $n_{1}$ is too small and the correction is positive. This method is very convenient when the numbers are so large as to require the use of a logarithm table in making the interpolation. For example, let it be required to find $\theta$ from Vega's ten-place table when log $\sin \theta=>.9107867247$.

The tabular values are as follows:-

\begin{tabular}{rcrc}
$\theta^{\prime}$ & $\log \sin \theta$. & \multicolumn{1}{c}{$\Delta^{\prime}}$. & $\Delta^{\prime \prime}$. \\
$4^{\circ} 40^{\prime \prime} 0^{\prime \prime}$ & 8.9104038653 & +2578594 & \\
10 & .9106617247 & 2577057 & -1537 \\
20 & .9109194304 & & -1538 \\
30 & .9111769823 & &
\end{tabular}

The quantity to be computed is

$$
\begin{gathered}
n=n_{1}-r b^{\prime} n_{1} . \\
* \quad \text { Loc. cit. p. } 195 .
\end{gathered}
$$


We disregard the algebraic sign of the last term, for a mere inspection of the first differences suffices to show that the correction to $n_{1}$ is positive. Following is the compulation :-

$$
\begin{aligned}
& \mathrm{F}_{n}=\quad 8.9107867247 \\
& F_{0}=\quad 8.9106617247 \\
& \Delta \mathrm{F}=\quad+1250000 \quad 6.0969100 \\
& a^{\prime}=a_{1}+b^{\prime}=\quad+2577825 \quad 6.4112534 \\
& n_{1}=\Delta \mathrm{F} \div a^{\prime}=\quad 0 \cdot 4849049 \quad 3 \cdot 6856566-10 \\
& r=n_{1} \div a^{\prime}=\quad 3 \cdot 2744-10 \\
& b^{\prime}=\frac{b_{0}}{2}=\quad-768 \quad 2.8854 \quad \text { Cheek. }
\end{aligned}
$$

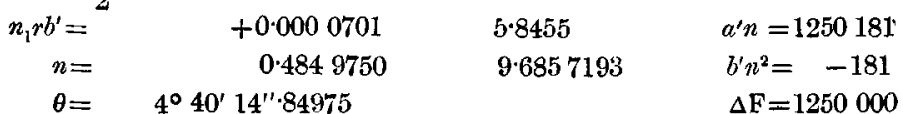

The value of the term $2 n_{1}\left(r b^{\prime}\right)^{2}$ is $2 \times 10^{-8}$, a negligible quantity. The formula here suggested will frequently be applicable when the differences are small numbers. The computation may be made by actual multiplication and division; but in general, logarithmic computation requires less labour. In either case, the method just explained is about as easy of application as any other, except that in miscellaneous computations, some care must be exercised to see that the term $2 n_{1}\left(r b^{\prime}\right)^{2}$ may be omitted. On the other hand if the higher differences need be taken into account, the complete formula has the important advantage of putting into evidence the terms necessary to obtain a required degree of accuracy. Thus, although third differences occur in that portion of Vega's ten-place table of the logarithmic sines for arguments $=$ or $>2^{\circ}$, the terms in $r c^{\prime}$ are negligible and the maximum value of $2 n_{1}\left(r b^{\prime}\right)^{2}$, which occurs in the immediate vicinity of $2^{\circ}$, is five units in the seventh decimal place. Consequently, the correction to the simple formula

$$
n=n_{1}-n_{1} r b^{\prime}
$$

is so small that it may be omitted except when extreme accuracy is required for arguments a little greater than $2^{\circ}$.

A problem of frequent occurrence in many branches of applied science is the reversion of an empirical formula expressed in the form of a power series. Since the relation is given in the form (i), it is only necessary to substitute in (3) to obtain the value of the argument or independent variable. As an illustration, find the temperature of quicksilver when its volume is 1.01825409 . The empirical formula given by Chappuis * expressing the increase of volume of

* Landolt,Bornstein Tabellen, p. 209. 
Interpolation by Means of a Reversed Series.

quicksilver as a function of the temperature, for temperatures ranging from $0^{\circ}$ to $100^{\circ} \mathrm{C}$., may be written

$$
\Delta v=\left[6 \cdot 259332_{-10}\right] t-\left[1 \cdot 470008_{-10}\right] t^{2}+\left[0 \cdot 059041_{-10}\right] t^{3} .
$$

Arranging the computation as before,

$$
\begin{array}{rlrl}
b^{\prime} & =1 \cdot 470008_{-10} & r b^{\prime} & =7 \cdot 212704_{n-10} \\
c^{\prime} & =0 \cdot 059041_{-10} & r c^{\prime} & =5 \cdot 801737_{-10} \\
\Delta \mathrm{F}=\Delta v & =8 \cdot 261360_{-10} & f_{1} & =7 \cdot 21412_{n} \\
a^{\prime} & =6 \cdot 259332_{-10} & f_{2} & =5 \cdot 80174_{-10} \\
n_{1}=t_{1} & =2 \cdot 002028 & t_{1} & =100 \cdot 4681 \\
r & =5 \cdot 742696 & f_{1} t_{1} & =+0 \cdot 1645 \\
& f_{2} t_{1}{ }^{2} & =-0 \cdot 6394 \\
t=\Sigma & =100^{\circ} \cdot 007 \mathrm{C}
\end{array}
$$

Instances may arise where it will be more convenient on account of the relative magnitude of the quantities to compute the coefficients of equation (2) instead of the auxiliary quantities $f_{1}, f_{2}, f_{3}, \ldots$ Written in this form, the inverse of Chappuis' equation is

$$
t=[3 \cdot 740668] \Delta v+[2 \cdot 692012](\Delta v)^{2}-[5 \cdot 021349](\Delta v)^{3} .
$$

Sometimes, however, the inverted series converges so slowly as to be useless. Probably the best manner of handling such tabulated data or empirical relations is to select or compute $n$ values of the function corresponding to $n$ values of the independent variable and substitute in an equation of the form,

$$
t=\mathrm{A}_{1}(\Delta v)+\mathrm{A}_{2}(\Delta v)^{2}+\mathrm{A}_{3}(\Delta v)^{3}+\ldots . \mathrm{A}_{n}(\Delta v)^{n},
$$

and then evaluate the $n$ unknowns $\left(A_{1}, A_{2}, \ldots A_{n}\right)$ by solving the $n$ linear equations. Proceeding in this manner and using the values

$$
\begin{array}{ll}
t=30^{\circ} \mathrm{C} . & \Delta v=\left[7 \cdot 736488_{-10}\right] \\
t=60^{\circ} \mathrm{C} . & \Delta v=\left[8 \cdot 038045_{-10}\right] \\
t=90^{\circ} \mathrm{C} . & \Delta v=\left[8 \cdot 215155_{-10}\right]
\end{array}
$$

there results for the last equation,

$$
t=[3 \cdot 740678] \Delta v+[2 \cdot 655719](\Delta v)^{2}-[5 \cdot 009834](\Delta v)^{3} \text {. }
$$

The coefficients of $(a)$ differ slightly from those of $(b)$. Substituting $\Delta v=0.0182541$, the values found for $t$ are

and

$$
\begin{aligned}
& t_{a}=100^{\circ} .007 \mathrm{C} ., \\
& t_{b}=99^{\circ} .999 \mathrm{C} .
\end{aligned}
$$

The value $t=100^{\circ} .007 \mathrm{C}$. is the same as found from equation (3), the modified form of the general formula for inverse interpolation. Either of these three methods is sufficiently accurate for this problem. The last two methods are 
to be used in preference to the first when a great number of values is to be interpolated. In the many instances in which only one or two values are to be obtained, the first method is the most convenient. Functions determined by any of the preceding methods may not of course be very reliable for extrapolation. The last formula, for example, is applicable between the limits $30^{\circ}$ and $90^{\circ}$, but is uncertain for values of $t<30^{\circ}$ and $>90^{\circ}$.

It is desirable to ascertain if some other arrangement of the terms of the reverted series would facilitate computation. The numerous possible combinations of the quantities are best exhibited by again making use of Professor McMahon's expression for the general term of a reverted series. Putting

$$
b_{1}=\frac{b^{\prime}}{\boldsymbol{a}^{\prime}} ; \quad b_{2}=\frac{c^{\prime}}{a^{\prime}} ; \quad b_{3}=\frac{d^{\prime}}{\boldsymbol{a}^{\prime}} ; \quad b_{4}=\frac{e^{\prime}}{a^{\prime}} ; \ldots,
$$

the $(m-1)$ th term of the reverted series may be written

$$
m(m+1)(m+2) \ldots(p+q+\ldots(m-2))\left[\Sigma \frac{b_{i}^{p}}{p:} \frac{b_{j}^{q}}{q !}\right] n^{m-1},
$$

in which the exponents and subscripts are subject to the condition

$$
p i+q j+\ldots=m-2 \text {. }
$$

Giving $m$ successive values, say from 3 to 8 , we easily find the terms containing all possible combinations of $b_{1}^{p} b_{2}^{q} \ldots b_{6}^{u}$. They are

$$
\begin{array}{rrrrrrrrrrrr}
m=3 & b_{1}, & & & & & & & \\
4 & b_{1}{ }^{2} & b_{2} & & & & & & & \\
5 & b_{1}{ }^{3} & b_{1} & b_{2} & b_{3} & & & & & & \\
6 & b_{1}{ }^{4} & b_{1}{ }^{2} b_{2} & b_{1} & b_{3} & b_{2} & b_{2} & b_{4} & & & & \\
7 & b_{1}{ }^{5} & b_{1}{ }^{3} & b_{2} & b_{1}{ }^{2} b_{3} & b_{3} b_{2} & b_{1} b_{4} & b_{5} & & & \\
8 & b_{1}{ }^{6} & b_{1}{ }^{4} & b_{2} & b_{1}{ }^{3} b_{3} & b_{4} b_{2} & b_{1}{ }^{2} b_{4} & b_{1} b_{5} & b_{1} b_{2} b_{3} & b_{3}{ }^{2} & b_{2}{ }^{3} & b_{6}
\end{array}
$$

Each term is of weight $m-2$. There are two combinations of the terms of nearly complete symmetry in which functions of the same quantities appear in a form suitable for computation. Thus, assuming that the given series terminates with the fifth power, one arrangement of the terms is

$$
\begin{aligned}
& +b_{1}+b_{1}{ }^{2}+b_{1}{ }^{3}+b_{1}{ }^{4}+b_{1}{ }^{3}+\ldots . \\
& +b_{2}\left[1+b_{1}+b_{1}{ }^{2}+b_{1}{ }^{3}+\ldots . .\right] \\
& +b_{3}\left[1+b_{1}+b_{1}{ }^{2}+b_{1}{ }^{3}+\ldots .\right] \\
& +b_{4}\left[1+b_{1}+b_{1}{ }^{2}+\ldots . .\right] \\
& +b_{5}\left[1+b_{1}+\ldots \ldots\right. \\
& +\ldots \ldots \ldots \ldots .
\end{aligned}
$$


and another is

$$
\begin{aligned}
& +b_{1}+b_{2}+b_{3}+b_{4}+\ldots . \\
& +b_{1}\left[b_{1}+b_{2}+b_{3}+\ldots\right] \\
& +b_{1}^{2}\left[b_{1}+b_{2}+b_{3}+\ldots .\right] \\
& +b_{1}^{3}\left[b_{1}+b_{2}+b_{3}+\ldots\right]+\ldots b_{2}\left[b_{2}+b_{3}+\ldots .\right]
\end{aligned}
$$

The elements have been selected firstly with respect to the verticals, and secondly with respect to the diagonals. Of other approximately homogeneous and symmetrical combinations there are none. Group (1) has been used in equation (3). Group (2) gives the formula

$$
\begin{aligned}
n=n_{1} & +\frac{b^{\prime}}{a^{\prime}} n_{1}^{2}\left[-1-\frac{c^{\prime}}{b^{\prime}} n_{1}-\frac{d^{\prime}}{b^{\prime}} n_{1}{ }^{2}-\frac{e^{\prime}}{b^{\prime}} n_{1}{ }^{3}-\ldots\right] \\
& +\left(\frac{b^{\prime}}{a^{\prime}}\right)^{2} n_{1}{ }^{3}\left[+2+5 \frac{c^{\prime}}{\bar{b}^{\prime}} n_{1}+6 \frac{d^{\prime}}{b^{\prime}} n_{1}{ }^{2}+\ldots+3\left(\frac{c^{\prime}}{b^{\prime}}\right)^{2} n_{1}{ }^{2}+\ldots\right] \\
& \left.+\left(\frac{b^{\prime}}{a^{\prime}}\right)^{3} n_{1}{ }^{4}\left[-5-21 \frac{c^{\prime}}{\bar{b}^{\prime}} n_{1}-\ldots\right]\right] \\
& +\left(\frac{b^{\prime}}{a^{\prime}}\right)^{4} n_{1}^{5}[+14+\ldots . .]
\end{aligned}
$$

or

$$
n=n_{1}+f_{1}\left(\frac{b^{\prime}}{a^{\prime}}\right) n_{1}{ }^{2}+f_{2}\left(\frac{b^{\prime}}{a^{\prime}}\right)^{2} n_{1}{ }^{3}+f_{3}\left(\frac{b^{\prime}}{a^{\prime}}\right)^{3} n_{1}{ }^{4}+f_{4}\left(\frac{b^{\prime}}{a^{\prime}}\right)^{4} n_{1}{ }^{5} \text {. . }
$$

As in equation (3), symmetry fails in the term $b_{2}{ }^{2} n_{1}{ }^{5}$. The functions within the brackets are not dependent upon a single quantity as in the other formula, but their evaluation involves only a summation of simple functions of the same variables. The distribution of algebraic signs is unique.

Computing again the interpolation interval $(n)$ from the data of the first problem, there results :

$$
\begin{array}{lr}
\log \left(\frac{b^{\prime}}{a^{\prime}}\right) n_{1}{ }^{2}=8 \cdot 004909_{-10} & n_{1}=+0.4326284 \\
\log \left(\frac{c^{\prime}}{b^{\prime}}\right) n_{1}{ }^{2}=8 \cdot 58290_{n-10} & f_{1}\left(\frac{b^{\prime}}{a^{\prime}}\right) n_{1}{ }^{2}=-0.0097313 \\
\log \left(\frac{c^{d}}{b^{\prime}}\right)^{2} n_{1}{ }^{2}=7 \cdot 16580_{-10} & f_{2}\left(\frac{b^{\prime}}{a^{\prime}}\right)^{2} n_{1}{ }^{3}=+0.0004293 \\
\log \left(\frac{d^{\prime}}{b^{\prime}}\right) n_{1}{ }^{2}=6.6565_{-10} & f_{3}\left(\frac{b^{\prime}}{a^{\prime}}\right)^{3} n_{1}{ }^{4}=-0.0000232 \\
\log \left(\frac{e^{\prime}}{b^{\prime}}\right) n_{1}{ }^{3}=4 \cdot 967_{-10} & f_{4}\left(\frac{b^{\prime}}{a^{\prime}}\right)^{4} n_{1}{ }^{5}=+0.0000018 \\
n=\Sigma & =+0.423305,
\end{array}
$$

the value obtained by the first method. 


\section{Mr. R. D. Kleeman on Different Kinds of $\gamma$ Rays}

The reversed series necessarily involves the quotients, powers, and products of the differences, and for this reason is difficult of computation. This difficulty is largely overcome in the above formulas by taking advantage of the homogeneity of the reverted series, and expressing certain factors, the $f$ 's, as functions of the same quantities. If the values of the $f$ 's, or preferably their logarithms, were tabulated, the labour involved in inverse interpolation would be not much greater than that of direct interpolation; for each of the terms in (4), (5), or (6) would then be given in the form of simple product expressions suitable for logarithmic computation. This method has the further advantage of being straightforward; and in so far as the reversion of the series is concerned, a check is provided without the introduction of any new quantities by the substitution of $n$ in equation (1).

The possibility of using the reversed series as a formula for inverse interpolation was suggested to me by Dr. George F. Becker of the U.S. Geological Survey, with whom I have cooperated in preparing a volume of mathematical tables * entitled 'Tables of Hyperbolic Functions,' and now in course of publication by the Smithsonian Institution of Washington, D.C. The formulas here suggested have been used to a certain extent in the preparation of these tables.

Washington, D.C.,

February 1908.

LIX. On the Different Kinds of $\gamma$ Rays of Radium, and the Secondary $\gamma$ Rays which they produce. By R. D. Kleeman, B.A., B.Sc., 1851 Exhibition Research Scholar of the University of Adelaide, and Research Student of Emmanuel College; Emmanuel College, Cambridge $\dagger$.

$\mathrm{T}$ a paper published in the Philosophical Magazine the writer showed that part of the $\gamma$ radiation of radium could be approximately divided into three groups of rays. Each of these groups of rays is selectively absorbed by one

* In this volume are tabulated the natural and logarithmic hyperbolic sines, cosines, tangents, and cotangents to five places; the nutural and logarithmic circular sines and cosines to fire places; the ascending and descending exponentjal to seven significant figures with $\log _{10} e^{\mu}$ to seven places; the natural logarithms of the integral numbers from 1 to 1000 to five places; the gudermannian to seven places and the corresponding angular equivalents; the anti-gudermannian to hundredths of a second; and other tables of minor importance. The arguments for the most part advance by ten-thousandths from 0 to 0.1 , by thousandths from 0.1 to $3 \cdot 0$, and by hundredths from $3 \cdot 0$ to 6.0 .

t Communicated by Prof. J. J. Thomson, F.R.S.

† Phil. Mag. Nov. 1907, p. 618. 